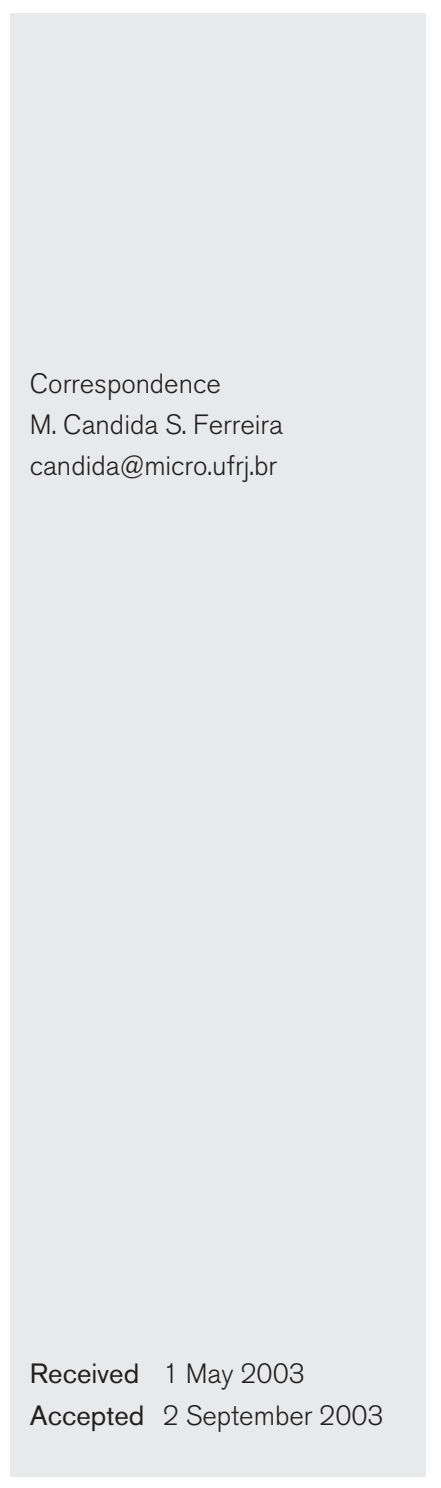

\section{Incidence and importance of Clostridium difficile in paediatric diarrhoea in Brazil}

\author{
Leandro J. F. Pinto, ${ }^{1}$ Ana P. P. Alcides, ${ }^{2}$ Eliane O. Ferreira, ${ }^{2}$ \\ Kátia E. S. Avelar, ${ }^{3}$ Aderbal Sabrá, ${ }^{4}$ Regina M. C. P. Domingues ${ }^{2}$ \\ and M. Candida S. Ferreira ${ }^{2}$ \\ ${ }^{1}$ Universidade Federal de Juiz de Fora, MG, Brazil \\ ${ }^{2}$ Instituto de Microbiologia Professor Paulo de Góes, UFRJ, RJ, Brazil \\ ${ }^{3}$ Instituto Oswaldo Cruz, Fiocruz, RJ, Brazil \\ ${ }^{4}$ Universidade do Grande Rio, RJ, Brazil
}

\begin{abstract}
Clostridium difficile strains were detected in 14 of 210 (6.7\%) faecal samples from children in Rio de Janeiro, Brazil, by cultivating faeces on cycloserine/cefoxitin/fructose agar after alcohol-shock. Two main groups of children were studied: inpatients $(n=96)$ and outpatients $(n=114)$. The inpatient group consisted of children on antibiotics or immunosuppressors who presented with diarrhoea and other children who did not present with diarrhoea and were not under an antibiotic or chemotherapeutic regimen. Among the outpatients, two groups were examined: namely, a group that comprised children who presented with diarrhoea and were occasionally under an antibiotic regimen and another group that comprised patients who were not taking antibiotics. After cytotoxic assay, toxigenic C. difficile $\left(\mathrm{Cd}\right.$ tox $\left.{ }^{+}\right)$strains were detected in $4.2 \%$ of inpatients and $3.5 \%$ of outpatients. Exclusion of other infectious causes of diarrhoea indicated a typical case of $C$. difficileassociated paediatric diarrhoea in the community. Among $\mathrm{Cd}$ tox ${ }^{+}$isolates, no variations were detected by PCR for toxin A that employed primers NK9 and NKVO11. No resistance was found to metronidazole or vancomycin among strains that were isolated from children who presented with diarrhoea, but the $\mathrm{MIC}_{50}$ and $\mathrm{MIC}_{90}$ values for clindamycin were 6-8 and $16 \mu \mathrm{g} \mathrm{ml}^{-1}$, respectively. Resistance to clindamycin seems to be more disseminated in strains from outpatients than in those from inpatients $(P<0.05)$. In conclusion, these data suggest that investigation for $C$. difficile infection should be taken into account in paediatric diarrhoea in both inpatients and outpatients in developing countries.
\end{abstract}

\section{INTRODUCTION}

Clostridium difficile has been recognized as the most important nosocomial pathogen in adults who manifest gastrointestinal symptoms subsequent to the use of broadspectrum antibiotics (Brazier, 2001). C. difficile has not usually been considered to be clinically important in stool specimens from neonates ( $<1$ month), as this organism can also be found as part of their normal gut microbiota (Rietra et al., 1978). However, in infants (between 1 month and 2 years) and older children ( $>2$ years), $C$. difficile colonization seems to become less frequent with increasing age, eventually reaching an isolation rate similar to that of an adult

Abbreviations: CDAD, Clostridium difficile-associated diarrhoea; ETEC, enterotoxigenic Escherichia coli; INCA, National Institute of Cancer; IPPMG, Instituto de Puericultura Professor Martagão Gesteira.
(McFarland et al., 2000). Nowadays, some authors also acknowledge that $C$. difficile can be an important cause of paediatric diarrhoea (McGowan \& Kader, 1999; McFarland et al., 2000). In such diarrhoea, the extent and degree of illness may seem to be worse in children than in adults, e.g. in fulminant enterocolitis (Price et al., 1988). Changes in the composition of the intestinal microbiota have been implicated in the initiation or maintenance of $C$. difficileassociated diarrhoea (CDAD), which occurs predominantly in patients whose colonic microbiota has been disrupted by antibiotic therapy (Hopkins \& MacFarlane, 2002). It has been established that the use of antibiotics by children presents the same risk as for adults. However, most literature in this field stems from the collection and interpretation of data from developed countries, where the use of antibiotics is under rigid control. Data collected in developing countries, on the other hand, can lead to a different interpretation, due to the widespread use of antibiotics. 
The purpose of this study was to evaluate the prevalence of toxigenic $C$. difficile strains in symptomatic outpatient and inpatient children. Antibiotic susceptibility levels of C. difficile strains were also assessed.

\section{METHODS}

Patients, stool samples and strains. We investigated the incidence rate of $C$. difficile strains (toxigenic or not) in the faeces of 210 children, aged between 3 months and 7 years, in the city of Rio de Janeiro, Brazil. Faecal samples were obtained from: (a) 114 outpatients, including 51 children with diarrhoea who were seen by doctors in several districts of Rio de Janeiro and were occasionally under an antibiotic regimen, 40 children without diarrhoea who were not using antibiotics and 23 children from day-care centres who did not present with diarrhoea; and (b) 96 inpatients, including 30 children with diarrhoea from the 'Instituto de Puericultura Professor Martagão Gesteira' (IPPMG) who were taking antibiotics, 49 neutropenic paediatric patients from the National Institute of Cancer (INCA) who were under a chemotherapeutic regimen and 17 inpatients from IPPMG who were taking antibiotics, but did not have diarrhoea. Specimens were collected after parental consent.

In 51 outpatients and 17 IPPMG patients with diarrhoea, other enteropathogens [enterotoxigenic Escherichia coli (ETEC), enteroaggregative E. coli (EAEC), enteropathogenic E. coli (EPEC), enterohaemorrhagic E. coli (EHEC), enteroinvasive E. coli (EIEC), Shiga toxin-producing E. coli (STEC), Salmonella sp., Shigella sp., Vibrio cholerae, Yersinia enterocolitica, Aeromonas sp., Campylobacter sp., enterotoxigenic Bacteroides fragilis, Plesiomonas sp., adenovirus, astrovirus, calicivirus, rotavirus and intestinal parasitic organisms] were investigated. Among INCA inpatients, there were no indications to investigate these other enteropathogens.

Diarrhoea was defined as three or more unformed stools in $24 \mathrm{~h}$. Information was obtained by using a standardized questionnaire that was submitted to the children's parents and by review of clinical charts. Outpatients who presented with diarrhoea were evaluated by primary care physicians in the community.

Two control strains were included in this investigation: C. difficile ATCC $9689^{\mathrm{T}}$ for culture in selective medium and cytotoxin and PCR analyses and Clostridium perfringens ATCC 10543 for susceptibility analysis.

Isolation and identification procedures. Stools were collected in sterile universal collectors and kept under refrigeration for no more than 18 h. Faecal samples were cultured directly or after enrichment (by using an alcohol-shock procedure) onto selective cycloserine/cefoxitin/ fructose agar and incubated anaerobically for $48 \mathrm{~h}$ at $37^{\circ} \mathrm{C}$ in jars filled with a gas mixture that consisted of $\mathrm{N}_{2}(80 \%), \mathrm{CO}_{2}(10 \%)$ and $\mathrm{H}_{2}$ $(10 \%)$. Several colonies (up to 10 ) from each patient were selected to investigate potential carriers of multiple strains. Isolates were identified as C. difficile by Gram-staining and standardized biochemical tests (Sumannen \& Baron, 1993).

\section{Toxin production}

Cytotoxin assay. To determine in vitro production of toxin $\mathrm{B}$, isolates were cultured for $72 \mathrm{~h}$ in brain heart infusion broth that had been prereduced and anaerobically sterilized (BHI-PRAS). Filtered $(0 \cdot 22 \mu \mathrm{m}$ membrane) supernatants $(0 \cdot 1 \mathrm{ml})$ were tested for cytotoxin in Vero monolayer cells added to $0.9 \mathrm{ml}$ Eagle's medium (Sigma) in 24-well microtitration plates. Plates were incubated for $24 \mathrm{~h}$ at $37^{\circ} \mathrm{C}$ under an atmosphere that contained $5 \% \mathrm{CO}_{2}$. C. difficile strains were considered to be toxin B-positive when $>50 \%$ of cells showed cell rounding.

Toxin A 'in situ'. Stool samples from outpatients with diarrhoea and neutropenic inpatients were evaluated for toxin A 'in situ' by a commercial enzyme immunoassay method (VIDAS CDA; Vitek).

\section{PCR assay to detect deletions of the repeating regions of the} toxin A gene

Genomic DNA extraction by using guanidine. The procedure of Pitcher et al. (1989) was followed. Cultures of toxin B-positive C. difficile strains grown in BHI-PRAS for $18 \mathrm{~h}$ were centrifuged at $8000 \mathrm{~g}$ for $5 \mathrm{~min}$. Supernatants were disposed and cells were washed with PBS $(\mathrm{pH} 7 \cdot 0)$. Cells were then treated with lysozyme $\left(50 \mathrm{mg} \mathrm{ml}^{-1}\right)$, resuspended in $100 \mu \mathrm{l} \mathrm{TE}$ (Tris, $10 \mathrm{mM}$; EDTA, $50 \mathrm{mM}$; pH 8.0) and incubated at $37^{\circ} \mathrm{C}$ for $30 \mathrm{~min}$. Afterwards, $5 \mathrm{M}$ guanidine isothiocyanate (Life Technologies) was added and the tubes were agitated and incubated at room temperature for $10 \mathrm{~min}$. Lysates were cooled on ice for $10 \mathrm{~min}$, then $7 \cdot 5 \mathrm{M}$ ammonium acetate was added and the mixture was kept on ice. Chloroform/isoamyl alcohol (24:1) was added, the DNA was precipitated and washed and any remaining ethanol was evaporated.

DNA primers and PCR procedure. The procedure of Kato et al. (1999) was followed. Briefly, $2 \mu \mathrm{l}$ DNA was added to $30 \mu \mathrm{l}$ reaction that contained $10 \mathrm{mM}$ Tris/HCl ( $\mathrm{pH} 8.3), 1.5 \mathrm{mM} \mathrm{MgCl}_{2}, 200 \mu \mathrm{M}$ each dNTP, 0.75 U Taq DNA polymerase (Life Technologies) and $4.5 \mathrm{ng}$ of each primer (NK9, 5'-CCACCAGCTGCAGCCATA-3'; NKV011, 5' TTTTGATCCTATAGAATCTAACTTAGTAAC-3'). Samples were amplified on a PE Applied Biosystems GeneAmp 9700 PCR system for 35 cycles of $95^{\circ} \mathrm{C}$ for $20 \mathrm{~s}, 60^{\circ} \mathrm{C}$ for $2 \mathrm{~min}$ and $74{ }^{\circ} \mathrm{C}$ for $5 \mathrm{~min}$. Amplification products were analysed by electrophoresis in $1.5 \%$ agarose gel on a horizontal gel electrophoresis apparatus (Horizon; Thistle Scientific). A molecular size marker (1 kb DNA Ladder; Life Technologies) was also used.

Antibiotic susceptibility by MIC determination. MICs for vancomycin, metronidazole and clindamycin were determined by Etest ( $A B$ Biodisk) for strains isolated from outpatients and by the agar dilution method (NCCLS, 1997) for strains isolated from inpatients.

Statistical analysis. Significance of differences in antibiotic susceptibility profiles was analysed by the $\chi^{2}$ test or by Fisher's exact two-tailed test with the Epi Info 6.04 software (Centers for Disease Control and Prevention).

\section{RESULTS}

C. difficile strains were isolated from 14 of 210 children $(6 \cdot 7 \%)$ among the different groups examined. Toxigenic $C$. difficile strains $\left(\mathrm{Cd}\right.$ tox $\left.^{+}\right)$were isolated from $4 \cdot 2 \%$ of inpatients and from $3.5 \%$ of outpatients (Table 1 ). Among children who harboured $C d$ tox ${ }^{+}$, especially those with diarrhoea as a symptom, concomitance of tox ${ }^{+}$and tox ${ }^{-}$ strains was detected in five patients. Exclusion of other infectious causes of diarrhoea among symptomatic outpatients contributed to the detection of a case of paediatric CDAD in subject 14LS (aged 3 years, 5 months) (Table 2). All strains that were positive by cytotoxic assay were also positive by PCR with primers NK9 and NKVO11, which yielded a PCR product of approximately $1200 \mathrm{bp}$ and therefore excluded any variations in the $t c d A$ gene; this identified all toxigenic $C$. difficile strains detected as positive for both toxin $\mathrm{A}$ and toxin $\mathrm{B}\left(\mathrm{A}^{+}, \mathrm{B}^{+}\right)$.

Table 3 shows the MIC ranges and the $\mathrm{MIC}_{50}$ and $\mathrm{MIC}_{90}$ values of metronidazole, vancomycin and clindamycin for 65 C. difficile isolates from children who presented with diar- 
Table 1. Recovery of Clostridium difficile strains from Brazilian children

\begin{tabular}{|c|c|c|c|c|c|c|c|}
\hline & \multicolumn{4}{|c|}{ Inpatients } & \multicolumn{3}{|c|}{ Outpatients } \\
\hline & Group 1 & Group 2 & Group 3 & Total & Group 1 & Group 2 & Total \\
\hline Intestinal disorders & Yes & Yes & No & & Yes & No & \\
\hline Positive cultures/no. stools tested & $2 / 30^{*}$ & $3 / 49 \dagger$ & $1 / 17 \ddagger$ & $6 / 96$ & $6 / 51 \S$ & $2 / 63 \|$ & $8 / 114$ \\
\hline \multicolumn{8}{|l|}{$C d$ tox $^{+}$in children aged: } \\
\hline$<24$ months & & & & & 1 & & \\
\hline$>24$ months & 1 & 3 & & 4 & 1 & 2 & 4 \\
\hline Percentage of $C d$ tox $^{+}$ & & & & $5 \cdot 2$ & & & $3 \cdot 5$ \\
\hline $\begin{array}{l}\text { Under antibiotic or chemotherapeutic } \\
\text { therapy\# }\end{array}$ & A & $\mathrm{C}$ & No & & $A^{* *}$ & No & \\
\hline
\end{tabular}

From ${ }^{*}, \dagger, \uparrow, \S$ and $\|$, respectively 7, 23, 5, 35 and 20 strains were isolated.

๑Cd tox ${ }^{+}$, toxigenic $C$. difficile strains.

\#A, Antibiotic; C, chemotherapeutic.

${ }^{* *}$ Occasionally.

Table 2. Characteristics of patients who harboured toxigenic Clostridium difficile strains

ND, Not detected; NE, not examined.

\begin{tabular}{|c|c|c|c|c|c|c|c|}
\hline Patient & Status & Diarrhoea $^{*}$ & $\begin{array}{c}\text { Antibiotic } \dagger(\mathrm{A}) \text { or } \\
\text { chemotherapeutic agent } \\
\text { (CA) }\end{array}$ & $\begin{array}{c}\text { Age } \\
\text { (months) }\end{array}$ & $\begin{array}{l}\text { Toxin A } \\
\text { 'in situ' }\end{array}$ & Other enteric pathogens & $\begin{array}{l}\text { No. strains } \\
\operatorname{Tox}^{+} / \text {Tox }\end{array}$ \\
\hline $14 \mathrm{LS}$ & Outpatient & Yes & A & 41 & Yes & $\mathrm{ND}$ & $1 / 4$ \\
\hline $4 \mathrm{LS}$ & Outpatient & Yes & No & 17 & Yes & Astrovirus, ETEC & $5 / 0$ \\
\hline IPd 6 & Inpatient & Yes & $\mathrm{A}$ & 84 & $\mathrm{NE}$ & $\mathrm{ND}$ & $4 / 0$ \\
\hline $11 \mathrm{D}$ & Outpatient & No & No & 28 & NE & $\mathrm{NE}$ & $1 / 0$ \\
\hline $12 \mathrm{LE}$ & Outpatient & No & No & 36 & No & $\mathrm{NE}$ & $4 / 2$ \\
\hline $9 \mathrm{LIN}$ & Inpatient & Yes & CA & $>24$ & Yes & $\mathrm{NE}$ & $1 / 7$ \\
\hline $12 \mathrm{LIN}$ & Inpatient & Yes & CA & $>24$ & Yes & $\mathrm{NE}$ & $7 / 1$ \\
\hline $21 \mathrm{LIN}$ & Inpatient & Yes & $\mathrm{CA}$ & $>24$ & Yes & $\mathrm{NE}$ & $2 / 5$ \\
\hline
\end{tabular}

${ }^{*}$ More than 10 loose watery stools per day.

$\uparrow$ Sulfametoxazole, trimethoprim, metronidazole.

rhoea. The antibiotics used for treatment of CDAD showed a narrow MIC range $\left(0 \cdot 047-2 \cdot 0\right.$ and $0 \cdot 25-1 \cdot 5 \mu \mathrm{gl}^{-1}$ for metronidazole and vancomycin, respectively), regardless of the origin of the isolates. $\mathrm{MIC}_{50}$ values for vancomycin $(0 \cdot 38$ and $1.0 \mu \mathrm{g} \mathrm{ml}^{-1}$ for isolates from outpatients and inpatients, respectively) and metronidazole $\left(0.094 \mu \mathrm{g} \mathrm{ml}^{-1}\right.$ for isolates from both outpatients and inpatients) were as low as the $\mathrm{MIC}_{90}$ values (respectively 0.19 and $1.0 \mu \mathrm{g} \mathrm{ml}^{-1}$ for metronidazole and 1.0 and $1.5 \mu \mathrm{g} \mathrm{ml}^{-1}$ for vancomycin). On the other hand, clindamycin showed a relatively broad MIC range $\left(3-16 \mu \mathrm{g} \mathrm{ml}^{-1}\right)$, notwithstanding the fact that the majority of isolates displayed similar sensitivity $\left(\mathrm{MIC}_{50}\right.$ and $\mathrm{MIC}_{90}$ values were $6-8$ and $16 \mu \mathrm{g} \mathrm{ml} \mathrm{m}^{-1}$, respectively). Among toxigenic strains, $63 \%$ of strains from outpatients and $28 \%$ of strains from inpatients were resistant to clindamycin, whereas resistance to clindamycin was detected in $70 \%$ of non-toxigenic strains from outpatients and in $52 \%$ of non-toxigenic strains from inpatients. These values revealed that strains from the community were more resistant to clindamycin $(P<0 \cdot 05)$.

\section{DISCUSSION}

According to the World Health Organization, the aetiologies of a great number of diarrhoeal illnesses remain unknown (Bern et al., 1992), which highlights the importance of monitoring the possible presence of novel pathogens.

C. difficile is widespread in nature and causes diarrhoea after the disruption of microbiota by antibiotic usage. The 
Table 3. Profile of susceptibility to clindamycin, metronidazole and vancomycin among Clostridium difficile strains isolated from paediatric outpatients $(n=35)$ and inpatients $(n=30)$ who presented with diarrhoea

\begin{tabular}{|lccc|}
\hline $\begin{array}{l}\text { Antimicrobial agent } \\
\text { (break-point) }\end{array}$ & \multicolumn{3}{c|}{ MIC $\left(\boldsymbol{\mu g ~ m l}{ }^{-1}\right)$} \\
\cline { 2 - 4 } & Range & MIC $_{50}$ & MIC $_{90}$ \\
\hline $\begin{array}{l}\text { Clindamycin }\left(8 \mu \mathrm{g} \mathrm{ml}^{-1}\right): \\
\quad \text { Outpatient }\end{array}$ & $4-16$ & 8 & 16 \\
$\quad$ Inpatient \\
$\begin{array}{l}\text { Metronidazole }\left(32 \mu \mathrm{g} \mathrm{ml}^{-1}\right): \\
\quad \text { Outpatient }\end{array}$ & $3-16$ & 6 & 16 \\
$\quad$ Inpatient \\
$\begin{array}{l}\text { Vancomycin }\left(4 \mu \mathrm{g} \mathrm{m}^{-1}\right): \\
\quad \begin{array}{l}\text { Outpatient } \\
\text { Inpatient }\end{array}\end{array}$ & $0 \cdot 047-0 \cdot 19$ & $0 \cdot 094$ & $0 \cdot 19$ \\
& $1-2$ & 1 & 1 \\
\hline
\end{tabular}

combination of self-medication and widespread use of overthe-counter drugs in countries where policies about medication are not strict might favour $C$. difficile colonization and, consequently, potential development of diarrhoea. Among the children examined in the present study, we detected a specific case of a toxigenic $C$. difficile strain present without any other enteropathogen in an outpatient child (aged 3 years, 5 months), which is characteristic of a true case of CDAD. A multicentre study carried out by our research group investigated enteropathogens that are prevalent in community diarrhoea and found that $C$. difficile was the third most frequently isolated species (Antunes et al., 2002). Riley et al. (1991) found that $C$. difficile was the agent isolated most frequently from community-acquired diarrhoea, which was probably a result of the dimension of the population studied (which included stools from adults and children). In our study, in the inpatient group, $\mathrm{Cd}$ tox ${ }^{+}$was isolated more often from children with intestinal disorders and recent antibiotic treatment than from carriers. Again, bearing in mind that CDAD in children has the same course as in adults or the elderly, colonic microbiota must be disturbed prior to C. difficile colonization. Cytotoxic drugs (aside from antibiotics) are prone to disturbing such colonic microbiota (Brazier \& Borriello, 2000). Hence, paediatric neutropenic patients who are undergoing cancer chemotherapy should also be monitored by bacteriological investigation in diarrhoeal diseases. Histopathological evidence of enterocolitis in such neutropenic patients fails frequently (Anand \& Glatt, 1993) and misdiagnosis can lead to complications in the treatment of these children. In our group of neutropenic patients, $6 \cdot 1 \%$ presented with both C. difficile tox ${ }^{+}$strains and toxin $\mathrm{A}$ in their faeces, which suggests an association of C. difficile with this group of patients. Therefore, investigation for toxigenic $C$. difficile in such patients should be maintained in our cancer unit.

Immunoenzymatic tests (ELISA) for toxin A, such as the one we performed in paediatric faecal specimens, may lead to misdiagnosis if this is the only type of test used to verify the presence of CDAD. On the other hand, toxin B detection by ELISA still has poor sensitivity and specificity (Kader et al., 1998). Detection by cytopathic assay or even through cultural methods is still appropriate in epidemiological investigations to identify and characterize strains (Brazier \& Borriello, 2000). It should be emphasized that the detection of phenotypic diversity of $C$. difficile strains in the same patient was due to cultural methods. By this procedure and by the larger number of colonies examined, it was possible to detect tox ${ }^{+}$and tox ${ }^{-}$C. difficile strains in the same patient.

With the aim of investigating C. difficile variant strains $\left(\mathrm{A}^{-}\right.$, $\mathrm{B}^{+}$), a PCR assay with primers specific to the $t c d A$ gene (Kato et al., 1999) was employed, but no variant strains were detected among paediatric isolates.

Sensitivity to vancomycin and metronidazole among the 65 strains studied confirms the susceptibility of $C$. difficile to these antibiotics, although some reports suggest an increasing emergence of strains with reduced susceptibility to metronidazole and vancomycin (Pelaez et al., 1998).

In spite of the fact that the break-points established for concentrations of drugs in serum are not usually achieved for intraluminal infections (Barbut et al., 1999), MIC data indicated that all isolates were susceptible to metronidazole and vancomycin. The two methodologies employed - Etest and agar dilution - have been shown to have good correlation for anaerobic bacteria, regardless of the antibiotic or species used (Citron et al., 1991; Wust \& Hardegger, 1992; Bolmström, 1993). Having said that, Barbut et al. (1999) have demonstrated that Etest results are always underestimations when compared to agar dilution data.

Even though clindamycin usage has decreased dramatically due to its induction of CDAD in the developed world, we do not have such information about developing countries. Selective pressure for clindamycin resistance seems to persist even in the community, as C. difficile strains isolated from outpatients (regardless of toxigenic activity) were more resistant to clindamycin than those from inpatients $(P<0 \cdot 05)$. The clinical impact of clindamycin resistance in the community should be further evaluated.

In conclusion, surveillance of paediatric diarrhoea in developing countries should take $C$. difficile into account in both inpatients and outpatients, especially when the symptoms are very pertinent and no other enteropathogen is detected.

\section{ACKNOWLEDGEMENTS}

This study was supported by grants from Faperj, Pronex, CNPq and Finep. We are grateful to Drs Angela Dantas, Anita Tibana, Eduardo Antunes, José Paulo Leite, Leila Campos, Marcia Guimarães, Maria Helena Aquino and Silvia Mondino for detection of other enteropathogens, Dr Maria Kadma Carriço, Laura Casquilha and Marcos Mota from INCA and the medical staff of Martagão Gesteira Pediatric Institute of the Federal University of Rio de Janeiro and Joaquim Santos Filho for technical assistance. 


\section{REFERENCES}

Anand, A. \& Glatt, A. E. (1993). Clostridium difficile infection associated with antineoplastic chemotherapy: a review. Clin Infect Dis 17, 109-113.

Antunes, E. N. F., Ferreira, E. O., Vallim, D. C., Paula, G. R., Seldin, L., Sabrá, A., Ferreira, M. C. S. \& Domingues, R. M. C. P. (2002). Pattern III non-toxigenic Bacteroides fragilis (NTBF) strains in Brazil. Anaerobe 8, $17-22$.

Barbut, F., Decré, D., Burghoffer, B. \& 8 other authors (1999). Antimicrobial susceptibilities and serogroups of clinical strains of Clostridium difficile isolated in France in 1991 and 1997. Antimicrob Agents Chemother 43, 2607-2611.

Bern, C., Martines, J., de Zoysa, I. \& Glass, R. I. (1992). The magnitude of the global problem of diarrhoeal disease: a ten-year update. Bull World Health Organ 70, 705-714.

Bolmström, A. (1993). Susceptibility testing of anaerobes with Etest. Clin Infect Dis 16 (Suppl. 4), S367-S370.

Brazier, J. S. (2001). Typing of Clostridium difficile. Clin Microbiol Infect 7, 428-431.

Brazier, J. S. \& Borriello, S. P. (2000). Microbiology, epidemiology and diagnosis of Clostridium difficile infection. Curr Top Microbiol Immunol 250, 1-33.

Citron, D. M., Ostovari, M. I., Karlsson, A. \& Goldstein, E. J. (1991). Evaluation of the E test for susceptibility testing of anaerobic bacteria. J Clin Microbiol 29, 2197-2203.

Hopkins, M. J. \& MacFarlane, G. T. (2002). Changes in predominant bacterial populations in human faeces with age and with Clostridium difficile infection. J Med Microbiol 51, 448-454.

Kader, H. A., Piccoli, D. A., Jaward, A. F., McGowan, K. L. \& Maller, E. S. (1998). Single toxin detection is inadequate to diagnose Clostridium difficile diarrhea in pediatric patients. Gastroenterology 115, 1329-1334.

Kato, H., Kato, N., Katow, S., Maegawa, T., Nakamura, S. \& Lyerly, D. M.
(1999). Deletions in the repeating sequences of the toxin A gene of toxin A-negative, toxin B-positive Clostridium difficile strains. FEMS Microbiol Lett 175, 197-203.

McFarland, L. V., Brandmarker, S. A. \& Guandalini, S. (2000). Pediatric Clostridium difficile: a phantom menace or clinical reality? J Pediatr Gastroenterol Nutr 31, 220-231.

McGowan, K. L. \& Kader, H. A. (1999). Clostridium difficile infection in children. Clin Microbiol Newsl 21, 49-53.

NCCLS (1997). Methods for Antimicrobial Susceptibility Testing of Anaerobic Bacteria, 4th edn. Approved Standard M11-A4. Villanova, PA: National Committee for Clinical Laboratory Standards.

Pelaez, T., Martinez-Sanchez, L., Alcala, L., Munoz, P., Garcia-Lechuz, J. M., Rodriguez-Creixems, M. \& Bouza, E. (1998). Metronidazole resistance in Clostridium difficile: a new emerging problem? In Program and Abstracts of the 38th Interscience Conference on Antimicrobial Agents and Chemotherapy, pp. 219, abstract no. E-173. Washington, DC: American Society for Microbiology.

Pitcher, D. G., Saunders, N. A. \& Owen, R. J. (1989). Rapid extraction of bacterial genomic DNA with guanidium thiocyanate. Lett Appl Microbiol 8, 151-156.

Price, E. M., Wright, V. M., Walker-Smith, J. A. \& Tabaqchali, S. (1988). Clostridium difficile and acute enterocolitis. Arch Dis Child 63, 543-545.

Rietra, P. J. G. M., Slaterus, K. W., Zanen, H. C. \& Meuwissen, S. G. M. (1978). Clostridial toxin in faeces of healthy infants. Lancet ii, 319.

Riley, T. V., Wetherall, F., Bowman, J., Mogyorosy, J. \& Golledge, C. L. (1991). Diarrhoeal disease due to Clostridium difficile in general practice. Pathology 23, 346-349.

Sumannen, P. \& Baron, E. J. (1993). Wadsworth Anaerobic Bacteriology Manual, 5th edn. Belmont, CA: Wadsworth.

Wust, J. \& Hardegger, U. (1992). Comparison of the E test and a reference agar dilution method for susceptibility testing of anaerobic bacteria. Eur J Clin Microbiol Infect Dis 11, 1169-1173. 